\title{
Hepatokine Selenoprotein P-Mediated Reductive Stress Causes Resistance to Intracellular Signal Transduction
}

\author{
Toshinari Takamura
}

\begin{abstract}
Significance: Selenoprotein $\mathrm{P}$ functions as a redox protein through its intrinsic thioredoxin domain and by distributing selenium to intracellular glutathione peroxidases, that is, glutathione peroxidase 1 and 4 .

Recent Advances: Selenoprotein P was rediscovered as a hepatokine that causes the pathology of type 2 diabetes and aging-related diseases, including exercise resistance in the skeletal muscle, insulin secretory failure in pancreatic $\beta$ cells, angiogenesis resistance in vascular endothelial cells, and myocardial ischemic-reperfusion injury. It was unexpected for the antioxidant selenoprotein $\mathrm{P}$ to cause insulin resistance, because oxidative stress associated with obesity and fatty liver is a causal factor for hepatic insulin resistance.

Critical Issues: Oxidative stress induced by the accumulation of reactive oxygen species (ROS) has a causal role in the development of insulin resistance, whereas ROS themselves function as intracellular second messengers that promote insulin signal transduction. ROS act both positively and negatively in insulin signaling depending on their concentrations. It might be possible that selenoprotein P causes "reductive stress" by eliminating a physiological ROS burst that is required for insulin signal transduction, thereby causing insulin resistance. In a large-scale intervention study, selenium supplementation that upregulates selenoprotein $\mathrm{P}$ was paradoxically associated with an increased risk for diabetes in humans. This review discusses the molecular mechanisms underlying the selenoprotein P-mediated resistance to angiogenesis and to exercise.

Future Directions: Selenoprotein P may be the first identified intrinsic factor that induces reductive stress, causing resistance to intracellular signal transduction, which may be the therapeutic target against sedentarylifestyle-associated diseases, such as diabetes and obesity. Antioxid. Redox Signal. 33, 517-524.

Keywords: diabetes, insulin resistance, oxidative stress, reductive stress, selenoprotein P, signal transduction

\section{Introduction}

$\mathbf{I}$ NSULIN RESISTANCE IS an underlying pathology in people with type 2 diabetes and its related vascular complications, cancers, and nonalcoholic fatty liver disease (NAFLD) (30). The liver, a major target organ of insulin, functions as a center to maintain whole-body energy homeostasis by sensing nutrient stimuli and by producing a variety of nutrients and bioactive substances (38). In an insulin-resistant state, impaired insulin action promotes hepatic glucose production, resulting in systemic hyperglycemia. Further, insulin resistance accelerates the pathology of NAFLD (30). On the other hand, ectopic fat accumulation

in the liver is associated with insulin resistance in the liver and skeletal muscle $(16,17)$. Therefore, it is indispensable to understand the molecular mechanisms underlying insulin signal transduction and its resistance, especially in hepatocytes.

Selenoprotein $\mathrm{P}$ was rediscovered as a diabetes-associated hepatokine that causes the pathology of type 2 diabetes (24). Findings related to selenoprotein $\mathrm{P}$ may help in understanding the significance of redox balance in fine-tuning biological signal transductions. In this review, we propose a concept of selenoprotein P-mediated "reductive stress" in signal transduction resistance that induces the pathology of type 2 diabetes and aging-related diseases.
\end{abstract}

Department of Endocrinology and Metabolism, Kanazawa University Graduate School of Medical Sciences, Kanazawa, Japan.

(C) Toshinari Takamura 2020; Published by Mary Ann Liebert, Inc. This Open Access article is distributed under the terms of the Creative Commons Attribution Noncommercial License (http://creativecommons.org/licenses/by-nc/4.0/) which permits any noncommercial use, distribution, and reproduction in any medium, provided the original author(s) and the source are cited. 


\section{Oxidative Stress Causes Insulin Resistance}

Chronic oxidative stress induced under the state of obesity or induced by toxic lipids such as free cholesterol (22) and the saturated fatty acid palmitate (27) may be an initial event that causes hepatic insulin resistance (21). Among the various causes of oxidative stress, the production of reactive oxygen species (ROS) by mitochondria or by nicotinamide adenine dinucleotide phosphate (NADPH) oxidase has a causal role in the development of insulin resistance (42). It has been reported that increased ROS levels trigger insulin resistance in cells treated with tumor necrosis factor $\alpha$ and glucocorticoids (12).

Specifically, in type 2 diabetes (23) and conditions of morbid obesity (37), increased levels of energy substrates such as glucose metabolism-derived acetyl-CoA and fatty acid metabolism-derived acyl-CoA are supplied into the mitochondria and used as substrates for oxidative phosphorylation (OXPHOS) to generate adenosine triphosphate (ATP). As hepatocytes uptake glucose independently of insulin, the excessive influx of glucose may easily activate intracellular OXPHOS in the diabetic condition. Similar to hepatocytes, a high-glucose condition activates OXPHOS in the mitochondria of endothelial cells (29), which transport glucose independently of insulin action. Mitochondrial OXPHOS is not only a major source of ATP but also a source of ROS production in the majority of cells. Electrons passing down the OXPHOS pathway "leak" from the major path and directly reduce oxygen molecules. Therefore, the increased mitochondrial oxygen flux results in the increased formation of ROS.

This excessive production of ROS is associated with the pathology of several human diseases. A C16:0 saturated fatty acid palmitate stimulates the generation of ROS that activates c-Jun $\mathrm{N}$-terminal kinase (JNK) during $\beta$ oxidation in the mitochondria (27). JNK activation causes insulin resistance in the liver, which is complicated with obesity-associated endoplasmic stress (31). Palmitate-induced production of ROS inhibits insulin signal transduction at the level of insulin receptor substrate-1 through JNK activation in hepatocytes (27). The upstream molecules that regulate JNK phosphorylation in response to the increases in cellular ROS levels include thioredoxin (TRX) and apoptosis signal-regulating kinase 1 (ASK1) (8). ROS oxidize TRX and, consequently, remove it from preexisting TRX-ASK1 complexes, resulting in the activation of ASK1 and JNK signaling (Fig. 1). A JNK inhibitor almost completely rescued cells from the palmitate-induced insulin resistance.

On the other hand, unexpectedly, the administration of antioxidants such as N-acetylcysteine (NAC) and $\alpha$ tocopherol even at maximum concentrations only partially rescued cells from the palmitate-induced insulin resistance, although it effectively suppressed the palmitate-induced activation of JNK (27). These findings suggest a paradox that antioxidants act as a double-edged sword in insulin action; therefore, the elimination of ROS using antioxidants may rescue the ROS-mediated insulin resistance and may exert a direct negative effect on insulin signaling. In fact, larger clinical intervention trials conducted to evaluate the potential of antioxidant supplements in preventing the development of diabetes could not observe any positive effects $(7,18,19)$.

Based on these conflicting findings, we hypothesized that excessive removal of ROS from cells does not necessarily ameliorate insulin resistance.

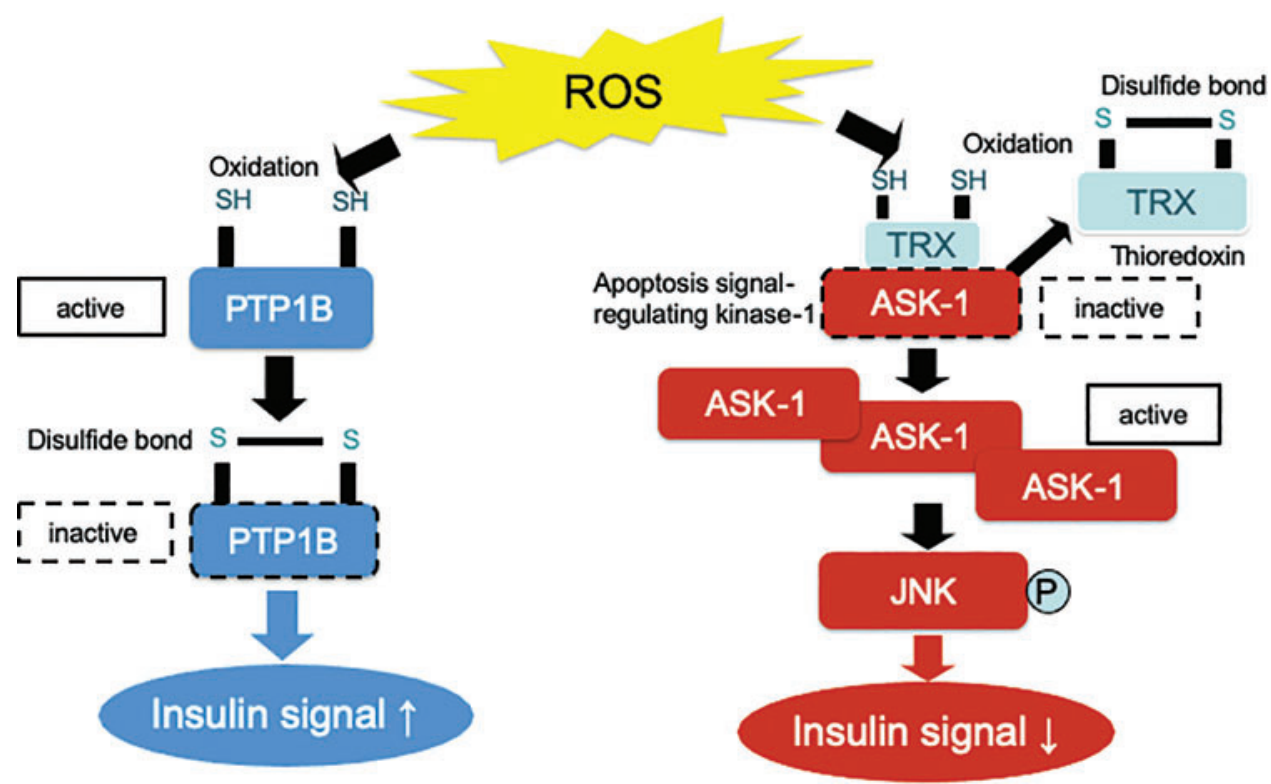

FIG. 1. Oxidation of thiol residues by ROS directly inhibits PTP1B activity. The structure of the active site and the low pKa of the thiol group of the invariant catalytic cysteine residue render PTP1B highly susceptible to reversible oxidation by $\mathrm{H}_{2} \mathrm{O}_{2}$. Oxidation of its active site cysteine residue abolishes the nucleophilic properties of PTP1B, causing its inactivation. PTP1B dephosphorylates the tyrosine residue of insulin receptor, resulting in the total inhibition of insulin signaling. On the other hand, ROS oxidizes TRX and, consequently, removes it from preexisting TRX-ASK1 complexes, leading to the activation of ASK1 and JNK signaling. It may be possible that the different sensitivity of thiol residue to $\mathrm{H}_{2} \mathrm{O}_{2}$ between PTP1B and TRX determines the insulin signaling. ASK1, apoptosis signal-regulating kinase 1; $\mathrm{H}_{2} \mathrm{O}_{2}$, hydrogen peroxide; JNK, c-Jun N-terminal kinase; PTP1B, protein tyrosine phosphatase 1B; ROS, reactive oxygen species; TRX, thioredoxin. Color images are available online. 


\section{Concentration-Dependent Dual Role of Oxidative Stress in Insulin Signal Transduction in Hepatocytes}

The molecular mechanisms underlying the paradoxical dual action of ROS in insulin signaling may involve intracellular compartments, timing, and concentration of ROS burst affecting insulin signaling. Low levels of ROS are required for normal cellular function and serve as second messengers to promote intracellular signal transduction by hormones such as insulin (10), angiotensin II (2), and vascular endothelial growth factor (VEGF). Such physiological levels of ROS are generated primarily at the site of the plasma membrane and endomembranes by NADPH oxidases (44). In an earlier study, Goldstein et al. demonstrated that insulin-induced in vitro endogenous hydrogen peroxide $\left(\mathrm{H}_{2} \mathrm{O}_{2}\right)$ enhances proximal and distal insulin signaling, at least partly through the oxidative inhibition of protein tyrosine phosphatase 1B (PTP1B) (10), which negatively regulates insulin action by dephosphorylating a tyrosine residue of the insulin receptor (9).

$\mathrm{H}_{2} \mathrm{O}_{2}$ is a representative membrane-permeable oxidant and the most abundant ROS in the cell (14). It has dual effects on insulin signal transduction in H4IIEC hepatocytes, and these roles depend on the $\mathrm{H}_{2} \mathrm{O}_{2}$ concentration used. Treatment with high concentrations $(10-25 \mu M)$ of $\mathrm{H}_{2} \mathrm{O}_{2}$ was found to reduce insulin-stimulated Akt phosphorylation by activating $\mathrm{JNK}$, whereas lower concentrations $(5-10 \mu M)$ of $\mathrm{H}_{2} \mathrm{O}_{2}$ rather enhanced this phosphorylation by suppressing PTP1B activity (14). These results are consistent with those of another previous report showing that blood concentrations of $\mathrm{H}_{2} \mathrm{O}_{2}$ are $\sim 1.7 \pm 2.5 \mu M$ in healthy humans (3). Therefore, depending on its concentration, and irrespective of the subcellular compartments in which it acts, $\mathrm{H}_{2} \mathrm{O}_{2}$ exerts a positive or negative effect on insulin signal transduction in hepatocytes. These findings suggest that the intracellular concentration of ROS themselves is a major factor in determining whether ROS impair or enhance insulin signaling.

The structure of the active site and the low pKa of the thiol group of the invariant catalytic cysteine residue render
PTP1B highly susceptible to reversible oxidation by $\mathrm{H}_{2} \mathrm{O}_{2}$ (1) (Fig. 1). Oxidation of its active site, the cysteine residue, abolishes the nucleophilic properties of PTP1B, resulting in its inactivation. PTP1B dephosphorylates the tyrosine residue of the insulin receptor, leading to the total inhibition of insulin signaling. Several types of phosphatases are known to inactivate the insulin signaling cascade by dephosphorylating different types of downstream effectors of insulin. Phosphatase and tensin homolog deleted on chromosome 10 (PTEN) dephosphorylates phosphatidylinositol 3, 4, 5-trisphosphate (PIP3) to terminate PI3 kinase signaling, resulting in the selective inhibition of PI3 kinase/Akt signaling. PTEN is also inactivated by $\mathrm{H}_{2} \mathrm{O}_{2}$-induced oxidation as well as by PTP1B (20).

It is known that $\mathrm{H}_{2} \mathrm{O}_{2}$ exerts both positive and negative effects on insulin signaling within a relatively narrow concentration range of 5-50 $\mu M$ (14). JNK and phosphatases such as PTP1B may be candidate molecules that determine the thresholds for the diametrical effects of $\mathrm{H}_{2} \mathrm{O}_{2}$ on cellular insulin signaling (Fig. 1). It may be possible that the action of $\mathrm{H}_{2} \mathrm{O}_{2}$ on cellular insulin signaling is determined by the net balance between the inactivation of PTP1B (enhancer for insulin signaling) and the activation of JNK (inhibitor for insulin signaling) (Fig. 1). For example, 10-50 $\mu M$ of $\mathrm{H}_{2} \mathrm{O}_{2}$ significantly decreased the activity of PTP1B, whereas these concentrations of $\mathrm{H}_{2} \mathrm{O}_{2}$ robustly increased the phosphorylation of JNK simultaneously (Fig. 2). As a result of the summation of these two opposite factors, insulin-induced Akt phosphorylation was impaired by $25-50 \mu M$ of $\mathrm{H}_{2} \mathrm{O}_{2}$, suggesting that the increase in JNK activation has a greater influence on net insulin signaling than the inactivation of PTP1B at these concentrations of $\mathrm{H}_{2} \mathrm{O}_{2}$. On the other hand, $5 \mu M$ of $\mathrm{H}_{2} \mathrm{O}_{2}$ induced a significant decrease in PTP1B activity without increasing JNK phosphorylation (Fig. 2), resulting in the enhancement of insulin-stimulated Akt phosphorylation. These results suggest that the decrease in PTP1B activity exerts a greater influence than the increase in JNK phosphorylation in cells treated with $5 \mu M$ of $\mathrm{H}_{2} \mathrm{O}_{2}$. Previous in vitro studies have
FIG. 2. Differential thresholds of PTP1B and JNK to ROS. Insulin signaling is balanced by the negative regulators PTP1B (A) and JNK (B). Low doses of $\mathrm{H}_{2} \mathrm{O}_{2}$ are sufficient to inhibit PTP1B activity (A), whereas high doses of $\mathrm{H}_{2} \mathrm{O}_{2}$ are required for JNK activation (B) (14). The different thresholds of PTP1B and JNK may cause the dual action of $\mathrm{H}_{2} \mathrm{O}_{2}$ in insulin signaling. Therefore, insulin signaling becomes enhanced under weak oxidative stress, whereas it is impaired under strong oxidative stress (14). $* * p<0.01$, versus untreated control. Color images are available online.
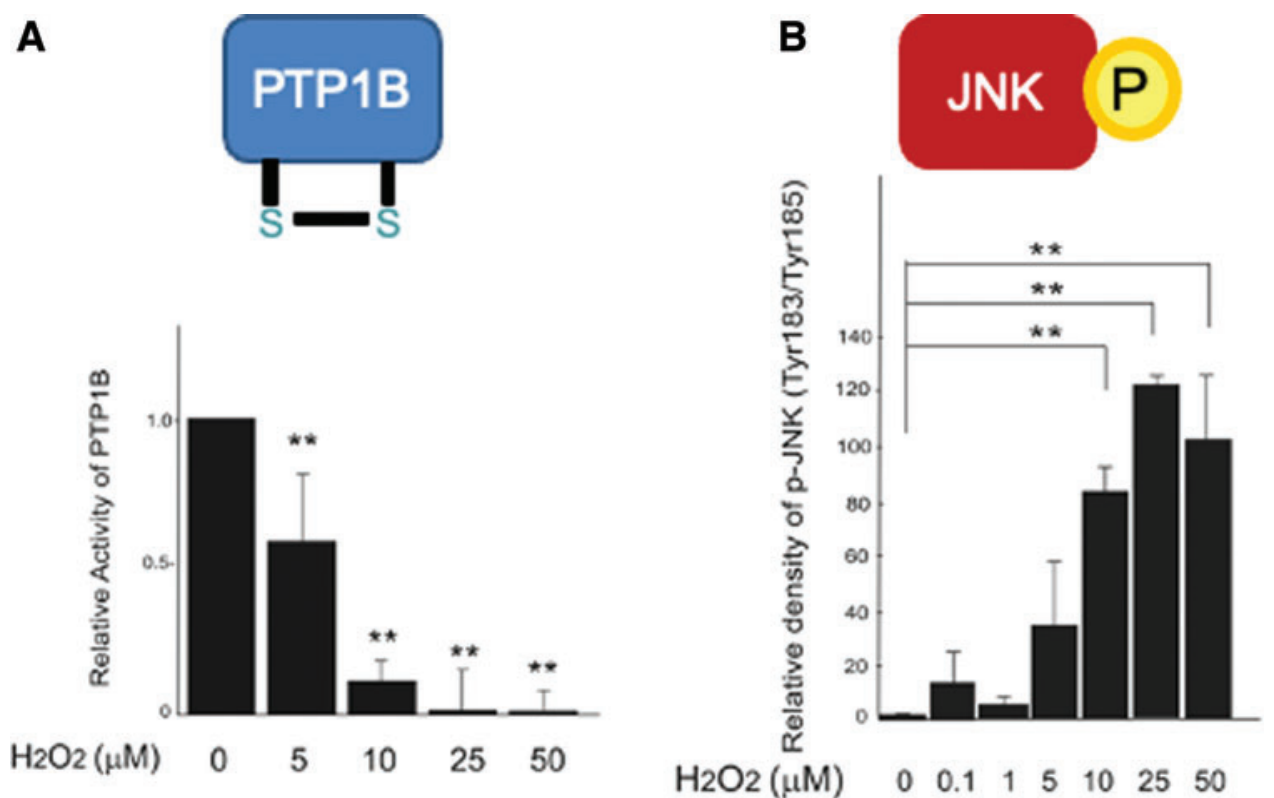
indicated that PTP1B may be $\sim 20$-fold more susceptible than TRX, an upstream regulator of JNK, to oxidation by $\mathrm{H}_{2} \mathrm{O}_{2}$ (46). Therefore, the dual actions of $\mathrm{H}_{2} \mathrm{O}_{2}$ in insulin signal transduction in hepatocytes may arise from the difference in susceptibility to $\mathrm{H}_{2} \mathrm{O}_{2}$ between PTP1B and TRX (Fig. 1).

Next, it was tested whether the antioxidant NAC attenuates the dual actions of $\mathrm{H}_{2} \mathrm{O}_{2}$ in insulin signal transduction (14). Pretreatment of H4IIEC hepatocytes with NAC at a concentration of $10 \mathrm{~m} M$, which corresponds to the maximum concentration of NAC commonly used in in vitro experiments, was sufficient to quench $\mathrm{H}_{2} \mathrm{O}_{2}$ at up to $50 \mathrm{mM}$. Moreover, NAC impaired the insulin-stimulated phosphorylation of Akt in the absence of $\mathrm{H}_{2} \mathrm{O}_{2}$. NAC also canceled the signal-enhancing action of low concentrations of $\mathrm{H}_{2} \mathrm{O}_{2}$. On the other hand, unexpectedly, NAC did not rescue the insulin resistance mediated by the high concentration of $\mathrm{H}_{2} \mathrm{O}_{2}$ (14), suggesting that excessive quenching of ROS by NAC rather impairs insulin signaling even in the pathological insulin resistance caused due to oxidative stress. Cells treated with $\mathrm{H}_{2} \mathrm{O}_{2}$ plus NAC showed lower levels of ROS than those in the absence of $\mathrm{H}_{2} \mathrm{O}_{2}$, suggesting that NAC at a concentration of $10 \mathrm{~m} M$ exerts an excessive potent antioxidative capacity. Therefore, pretreatment with NAC inhibits insulin signaling generally by attenuating the insulin-induced production of endogenous $\mathrm{H}_{2} \mathrm{O}_{2}$ required for the suppression of phosphatase activity due to its strong antioxidative capacity.

\section{Selenoprotein $\mathbf{P}$ as a Hepatokine That Causes the Pathology of Type 2 Diabetes}

The liver is a control center for systemic nutrient homeostasis. The liver functions as a center to maintain whole-body energy homeostasis by sensing nutrient stimuli and by producing a variety of nutrients and bioactive substances. Based on the fact that fat accumulation in the liver is associated with insulin resistance in the skeletal muscle, we hypothesized that a liver-derived hormone, hepatokine, affects the insulin sensitivity in distant organs. Information about human hepatic gene expression accumulated using serial analysis of gene expression technique (39) and DNA chip methods (23,
$37,40)$ was used to identify genes with signal peptides whose hepatic expression levels significantly correlated with glycemic control, obesity, or insulin resistance. Expression of the candidate hepatokine genes was further evaluated in various animal models of diabetes, obesity, and fatty liver. Based on the results of these approaches, we isolated 62 candidate genes for hepatokines associated with insulin resistance, hyperglycemia, and obesity.

Of these candidates, the SELENOP gene encoding selenoprotein $\mathrm{P}$ was identified as a gene whose expression levels positively correlated with insulin resistance and hyperglycemia (24). In fact, serum levels of selenoprotein P were elevated in people with type 2 diabetes and significantly correlated with fasting plasma glucose and HbA1c levels (24). It has also been reported that selenoprotein $\mathrm{P}$ is the most abundant selenoprotein in plasma and is primarily produced in the liver. The selenoprotein $\mathrm{P}$ molecule contains 10 selenocysteine residues per molecule $(4,33)$. SELENOP is upregulated by selenium supply, followed by incorporation into selenocysteine. The expression of SELENOP is also regulated through the transcription factors FoxOs and SREBP-1 (Fig. 3).

Studies have shown that insulin downregulated SELENOP by phosphorylating and inactivating FoxO1 (34), whereas the antidiabetic drug metformin activated adenosine monophosphate-activated protein kinase (AMPK), phosphorylated and inactivated FoxO3a but not FoxO1, and thereby downregulated SELENOP in hepatocytes (41). Eicosapentaenoic acid, one of the $\omega-3$ polyunsaturated fatty acids, downregulates SELENOP by inhibiting nuclear transport and promoter binding of SREBP-1c (36) (Fig. 3).

It is known that selenoprotein $\mathrm{P}$ functions as a selenium transporter protein from the liver to the peripheral organs and as an antioxidant protein (4). However, its role in energy metabolism has still remained unknown. An earlier study showed that mice in which the antioxidant hepatokine selenoprotein $\mathrm{P}$ was deleted were protected from high-fat-dietinduced insulin resistance in the liver and skeletal muscle (24). It has been reported that selenoprotein P causes insulin resistance in the liver, at least in part, by inactivating AMPK

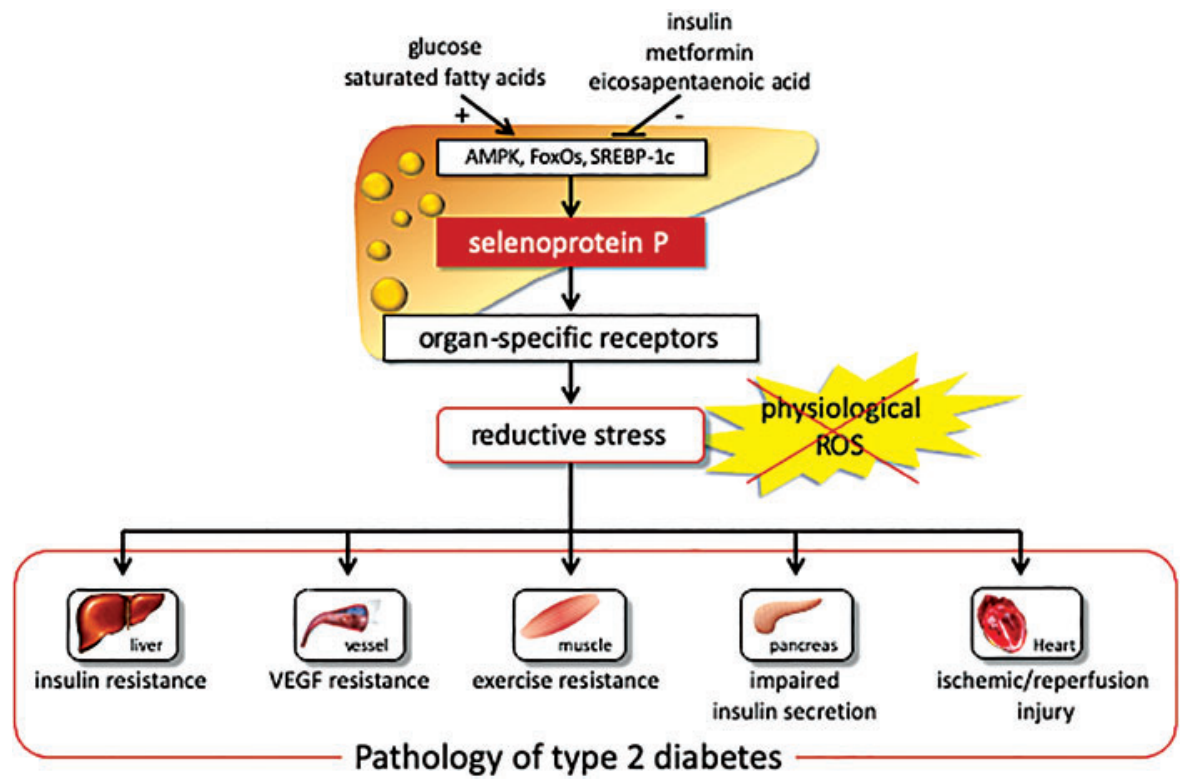

FIG. 3. Selenoprotein $P$ causes the pathology of type 2 diabetes through reductive stress. Selenoprotein $\mathrm{P}$ expression is regulated positively with glucose and negatively with insulin. Selenoprotein $\mathrm{P}$ causes insulin resistance in the liver (24), exercise resistance in the skeletal muscle (25), insulin secretory failure in $\beta$ cells (26), VEGF resistance in vascular endothelial cells (13), and myocardial ischemic-reperfusion injury via IGF-1 resistance (6), all of which are involved in diabetic complications and aging-related diseases. IGF-1, insulin-like growth factor-1; VEGF, vascular endothelial growth factor. Color images are available online. 
FIG. 4. Selenoprotein $P$ causes VEGF resistance and impairs vasculogenesis through reductive stress. In HUVECs, ROS burst is required for VEGF signal transduction by shutting down phosphatase PTP1B. Selenoprotein P scavenges the ROS burst required for signal transduction and thereby induces "VEGF resistance" in endothelial cells (13). HUVECs, human umbilical vein endothelial cells. Color images are available online.

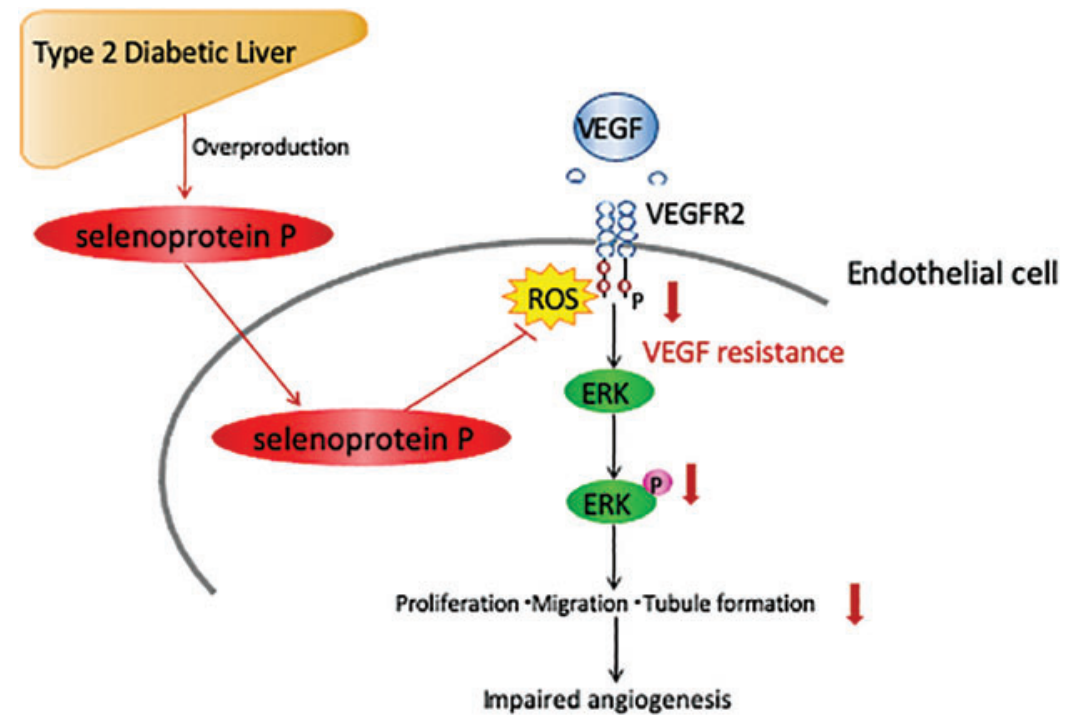

(24). Selenoprotein $P$ also induces exercise resistance in the skeletal muscle (25), impairs insulin secretion from $\beta$ cells (26), impairs angiogenesis by inducing VEGF resistance in vascular endothelial cells (13), and enhances myocardial ischemic-reperfusion injury through insulin-like growth factor-1 resistance (6), all of which are involved in diabetic complications and aging-related diseases (Fig. 4).

\section{Selenoprotein P Causes Signal Transduction Resistance Through Reductive Stress}

In previous studies, it was an unexpected finding that the antioxidant selenoprotein $P$ causes insulin resistance because oxidative stress associated with obesity and fatty liver was a well-recognized causal factor for hepatic insulin resistance (21, 22, 27). Again, selenoprotein $P$ functions as a redox protein through its intrinsic TRX domain and by distributing selenium to the intracellular glutathione peroxidases glutathione peroxidase (Gpx1) 1 and $4(4,33)$. In fact, mice lacking Gpx 1, a key cytosolic enzyme involved in the removal of ROS, were protected from high-fat diet-induced insulin resistance (20). These findings provide causal evidence for the enhancement of insulin signaling by ROS in vivo. It might be possible that selenoprotein $\mathrm{P}$ eliminates a physiological ROS burst that is required for insulin signal transduction and thereby causes insulin resistance, the condition referred to as reductive stress. In fact, large-scale intervention studies and observation studies have demonstrated that selenium supplementation that upregulates selenoprotein $\mathrm{P}$ was paradoxically associated with an increased risk for diabetes in humans $(35,45)$.

Impaired angiogenesis induced by VEGF resistance is a hallmark of the vascular complications of type 2 diabetes. Selenoprotein $\mathrm{P}$ impairs angiogenesis/vasculogenesis through resistance to VEGF (13) angiogenesis (Fig. 4). Specifically, physiological concentrations of selenoprotein $\mathrm{P}$ inhibit the VEGF-stimulated cell proliferation, tubule formation, and migration in human umbilical vein endothelial cells (HUVECs). Wound closure was impaired in mice overexpressing $S E$ LENOP, whereas it was improved in SELENOP-/- mice. SELENOP-/- mice displayed an increase in blood flow recovery and vascular endothelial cells after hindlimb ischemia.
It was observed that VEGF binding to VEGF receptor 2 (VEGFR2) stimulated NADPH oxidase to generate ROS at the site of the plasma membrane in endothelial cells (43). This transient ROS burst oxidizes and inactivates protein tyrosine phosphatases, which negatively regulate VEGF signaling and thereby promote VEGFR2 phosphorylation and the subsequent signaling cascade (43). Selenoprotein P suppresses the VEGF-induced generation of ROS and the phosphorylation of VEGFR2 and extracellular signal-regulated kinase $1 / 2$ in HUVECs (13). These data suggest that selenoprotein P exerts reductive stress that negatively regulates VEGF signaling and thereby inhibits angiogenesis (Fig. 4).

Another example of the selenoprotein P-mediated reductive stress is the exercise resistance (Fig. 5). It is known that physical exercise has health-promoting effects in humans. However, its effects on enhanced endurance capacity vary among individuals. Specifically, people with type 2 diabetes tend to exhibit a poor response to exercise in hypoglycemia, which limits the benefits obtained from the health-promoting effects of physical exercise. It has been believed that such exercise resistance is determined by genetically and/or epigenetically altering the expression of the genes involved in skeletal muscle biology and exercise effects such as peroxisome proliferative activated receptor $\gamma$ coactivator (PGC)- $1 \alpha$, peroxisome proliferator-activated receptor $\beta / \delta$, and pyruvate dehydrogenase kinase 4 . We found that mice with SELENOP deletion exhibited the "super-endurance" phenotype, which is characterized by increased exercise sensitivity and endurance capacity, through the upregulation of ROS that phosphorylates AMPK in the skeletal muscle (Fig. 5). Downstream of PGC- $1 \alpha$, the mRNA levels of mitochondria-related genes and genes encoding antioxidative enzymes such as uncoupling protein 3 (Ucp3), estrogen-related receptor alpha (Essra), catalase $(\mathrm{Cat})$, and mitochondrial superoxide dismutase 2 (Sod2) were upregulated in exercised SELENOP-deficient mice compared with those in exercised wild-type mice.

Exercise-induced generation of ROS not only causes oxidative damage but also functions as signaling molecules mediating beneficial molecular adaptations (28). AMPK is activated by acute exposure to ROS (5) and upregulates PGC$1 \alpha$, which has a major role in exercise-mediated muscle 


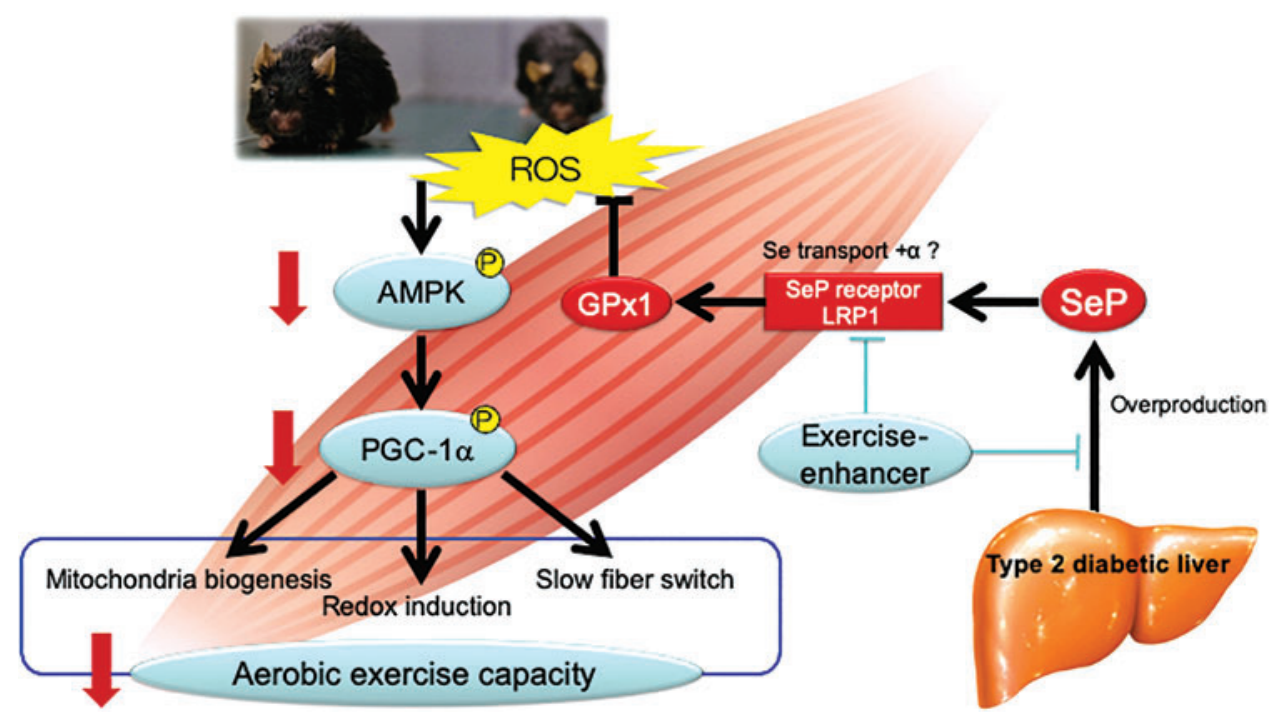

FIG. 5. Exercise resistance caused by selenoprotein $\mathbf{P}$-mediated reductive stress. Exercise generates ROS, activates AMPK, and thereby activates PGC- $1 \alpha$ leading to mitochondrial biogenesis, redox induction, and slow fiber switch. Overproduced selenoprotein $\mathrm{P}$ is transported via its receptor LRP-1, which is one of the LDL receptor family members, eliminates exercise-induced ROS, and cancels aerobic exercise capacity (25). Such reductive stress may be a causal factor for "exercise resistance" in diabetes. Inhibiting the production or receptor binding of selenoprotein P may be the therapeutic target to enhance exercise in people with diabetes. AMPK, adenosine monophosphate-activated protein kinase; LDL, low-density lipoprotein; LRP-1, low density lipoprotein receptor-related protein 1; PGC, peroxisome proliferative activated receptor $\gamma$ coactivator. Color images are available online.

adaptations (15). Therefore, the exercise-mediated generation of ROS may mediate the effects of exercise training on activating AMPK and PGC- $1 \alpha$ in the skeletal muscle. It may be possible that increased levels of selenoprotein $\mathrm{P}$ in the diabetic state eliminate the exercise-evoked generation of ROS and thereby diminish the health-promoting effects of exercise. In fact, supplementation with the antioxidant NAC was found to cancel the exercise-induced production of ROS and reduce endurance capacity after chronic exercise in SELENOP-deficient mice (25). In cultured C2C12 myotubes, selenoprotein $\mathrm{P}$ treatment impairs the ROS-induced AMPK phosphorylation. Selenoprotein $\mathrm{P}$ also reduces the mRNA levels of Ppargcla gene, which are canceled with the knockdown of AMPK $\alpha 1$ and 2 (25). In addition, increased levels of circulating selenoprotein $\mathrm{P}$ can predict the ineffectiveness of training on endurance capacity in humans (25). Indeed, supplementation with antioxidants such as vitamin $\mathrm{C}$ and vitamin $\mathrm{E}$ was found to abolish the exercise-induced molecular alterations and limit the positive effects of exercise training on endurance capacity in humans $(11,32)$.

These findings suggest that adequate oxidative stress is required for the health-promoting effects of exercise. Selenoprotein $\mathrm{P}$ weakens the exercise-evoked activation of AMPK and thereby hampers the effects of exercise on endurance capacity through reductive stress (Fig. 5). Therefore, selenoprotein $\mathrm{P}$ may be the first identified intrinsic factor that renders reductive stress, causing exercise resistance, which may be the therapeutic target against diseases associated with a sedentary lifestyle such as diabetes and obesity.

\section{Acknowledgments}

The author thanks Kiyo-aki Ishii, Hiroaki Takayama, Hirofumi Misu, and Yoshiro Saito for useful discussions.

\section{Funding Information}

This study was partly supported by JSPS KAKENHI Grant Nos. JP17H04199 and JP18K19560 and the Takeda Science Foundation.

\section{References}

1. Andersen JN, Mortensen $\mathrm{OH}$, Peters GH, Drake PG, Iversen LF, Olsen OH, Jansen PG, Andersen HS, Tonks NK, and Møller NP. Structural and evolutionary relationships among protein tyrosine phosphatase domains. Mol Cell Biol 21: 7117-7136, 2001.

2. Anilkumar N, Weber R, Zhang M, Brewer A, and Shah AM. Nox4 and Nox 2 NADPH oxidases mediate distinct cellular redox signaling responses to agonist stimulation. Arterioscler Thromb Vasc Biol 28: 1347-1354, 2008.

3. Banerjee D, Madhusoodanan UK, Sharanabasappa M, Ghosh S, and Jacob J. Measurement of plasma hydroperoxide concentration by FOX-1 assay in conjunction with triphenylphosphine. Clin Chim Acta 337: 147-152, 2003.

4. Burk RF and Hill KE. Selenoprotein P: an extracellular protein with unique physical characteristics and a role in selenium homeostasis. Annu Rev Nutr 25: 215-235, 2005.

5. Cardaci S, Filomeni G, and Ciriolo MR. Redox implications of AMPK-mediated signal transduction beyond energetic clues. J Cell Sci 125: 2115-2125, 2012.

6. Chadani H, Usui S, Inoue O, Kusayama T, Takashima S, Kato T, Murai H, Furusho H, Nomura A, Misu H, Takamura T, Kaneko S, and Takamura M. Endogenous selenoprotein $\mathrm{P}$, a liver-derived secretory protein, mediates myocardial ischemia/reperfusion injury in mice. Int $\mathrm{J} \mathrm{Mol}$ Sci 19: 878, 2018.

7. Czernichow S, Couthouis A, Bertrais S, Vergnaud A-C, Dauchet L, Galan P, and Hercberg S. Antioxidant supplementation does not affect fasting plasma glucose in the 
Supplementation with Antioxidant Vitamins and Minerals (SU.VI.MAX) study in France: association with dietary intake and plasma concentrations 1-3. Am J Clin Nutr 84: 395-399, 2006.

8. Fujino G, Noguchi T, Takeda K, and Ichijo H. Thioredoxin and protein kinases in redox signaling. Semin Cancer Biol 16: 427-435, 2006.

9. Goldstein BJ. Protein-tyrosine phosphatase 1B (PTP1B): a novel therapeutic target for type 2 diabetes mellitus, obesity and related states of insulin resistance. Curr Drug Targets Immune Endocr Metabol Disord 1: 265-275, 2001.

10. Goldstein BJ, Mahadev K, Wu X, Zhu L, and Motoshima $\mathrm{H}$. Role of insulin-induced reactive oxygen species in the insulin signaling pathway. Antioxid Redox Signal 7: 10211031, 2005.

11. Gomez-Cabrera M-C, Domenech E, Romagnoli M, Arduini A, Borras C, Pallardo FV, Sastre J, and Viña J. Oral administration of vitamin $\mathrm{C}$ decreases muscle mitochondrial biogenesis and hampers training-induced adaptations in endurance performance. Am J Clin Nutr 87: 142-149, 2008.

12. Houstis N, Rosen ED, and Lander ES. Reactive oxygen species have a causal role in multiple forms of insulin resistance. Nature 440: 944-948, 2006.

13. Ishikura K, Misu H, Kumazaki M, Takayama H, MatsuzawaNagata N, Tajima N, Chikamoto K, Lan F, Ando H, Ota T, Sakurai M, Takeshita Y, Kato K, Fujimura A, Miyamoto K-II, Saito Y, Kameo S, Okamoto Y, Takuwa Y, Takahashi K, Kidoya H, Takakura N, Kaneko S, and Takamura T. Selenoprotein $\mathrm{P}$ as a diabetes-associated hepatokine that impairs angiogenesis by inducing VEGF resistance in vascular endothelial cells. Diabetologia 57: 1968-1976, 2014.

14. Iwakami S, Misu H, Takeda T, Sugimori M, Matsugo S, Kaneko S, and Takamura T. Concentration-dependent dual effects of hydrogen peroxide on insulin signal transduction in h4iiec hepatocytes. PLoS One 6: e27401, 2011.

15. Kang C, O'Moore KM, Dickman JR, and Ji LL. Exercise activation of muscle peroxisome proliferator-activated receptor $-\gamma$ coactivator- $1 \alpha$ signaling is redox sensitive. Free Radic Biol Med 47: 1394-1400, 2009.

16. Kato K-I, Takeshita Y, Misu H, Zen Y, Kaneko S, and Takamura T. Liver steatosis is associated with insulin resistance in skeletal muscle rather than in the liver in Japanese patients with non-alcoholic fatty liver disease. $J$ Diabetes Investig 6: 158-163, 2015.

17. Kato K, Takamura T, Takeshita Y, Ryu Y, Misu H, Ota T, Tokuyama K, Nagasaka S, Matsuhisa M, Matsui O, and Kaneko S. Ectopic fat accumulation and distant organspecific insulin resistance in japanese people with nonalcoholic fatty liver disease. PLoS One 9: e92170, 2014.

18. Liu S, Ajani U, Chae C, Hennekens C, Buring JE, and Manson JE. Long-term $\beta$-Carotene supplementation and risk of type 2 diabetes mellitus. JAMA 282: 1073-1075, 1999.

19. Liu S, Lee I-M, Song Y, Van Denburgh M, Cook NR, Manson JE, and Buring JE. Vitamin E and risk of type 2 diabetes in the women's health study randomized controlled trial. Diabetes 55: 2856-2862, 2006.

20. Loh K, Deng H, Fukushima A, Cai X, Boivin B, Galic S, Bruce C, Shields BJ, Skiba B, Ooms LM, Stepto N, Wu B, Mitchell CA, Tonks NK, Watt MJ, Febbraio MA, Crack PJ, Andrikopoulos S, and Tiganis T. Reactive oxygen species enhance insulin sensitivity. Cell Metab 10: 260-272, 2009.

21. Matsuzawa-Nagata N, Takamura T, Ando H, Nakamura S, Kurita S, Misu H, Ota T, Yokoyama M, Honda M, Miya- moto K, and Kaneko S. Increased oxidative stress precedes the onset of high-fat diet-induced insulin resistance and obesity. Metabolism 57: 1071-1077, 2008.

22. Matsuzawa N, Takamura T, Kurita S, Misu H, Ota T, Ando H, Yokoyama M, Honda M, Zen Y, Nakanuma Y, Miyamoto $\mathrm{K}$, and Kaneko S. Lipid-induced oxidative stress causes steatohepatitis in mice fed an atherogenic diet. $\mathrm{He}$ patology 46: 1392-1403, 2007.

23. Misu H, Takamura T, Matsuzawa N, and Shimizu A. Genes involved in oxidative phosphorylation are coordinately upregulated with fasting hyperglycaemia in livers of patients with type 2 diabetes. Diabetologia 50: 268-277, 2007.

24. Misu H, Takamura T, Takayama H, Hayashi H, MatsuzawaNagata N, Kurita S, Ishikura K, Ando H, Takeshita Y, Ota T, Sakurai M, Yamashita T, Mizukoshi E, Yamashita T, Honda M, Miyamoto KI, Kubota T, Kubota N, Kadowaki T, Kim HJ, Lee IK, Minokoshi Y, Saito Y, Takahashi K, Yamada Y, Takakura N, and Kaneko S. A liver-derived secretory protein, selenoprotein $\mathrm{P}$, causes insulin resistance. Cell Metab 12: 483-495, 2010.

25. Misu H, Takayama H, Saito Y, Mita Y, Kikuchi A, Ishii K, Chikamoto K, Kanamori T, Tajima N, Lan F, Takeshita Y, Honda M, Tanaka M, Kato S, Matsuyama N, Yoshioka Y, Iwayama K, Tokuyama K, Akazawa N, Maeda S, Takekoshi K, Matsugo S, Noguchi N, Kaneko $\mathrm{S}$, and Takamura T. Deficiency of the hepatokine selenoprotein $\mathrm{P}$ increases responsiveness to exercise in mice through upregulation of reactive oxygen species and AMP-activated protein kinase in muscle. Nat Med 23: 508-516, 2017.

26. Mita Y, Nakayama K, Inari S, Nishito Y, Yoshioka Y, Sakai N, Sotani K, Nagamura T, Kuzuhara Y, Inagaki K, Iwasaki M, Misu H, Ikegawa M, Takamura T, Noguchi $\mathrm{N}$, and Saito Y. Selenoprotein P-neutralizing antibodies improve insulin secretion and glucose sensitivity in type 2 diabetes mouse models. Nat Commun 8: 1658, 2017.

27. Nakamura S, Takamura T, Matsuzawa-nagata N, Takayama H, Misu H, Noda H, Nabemoto S, Kurita S, Ota T, Ando H, Miyamoto K, and Kaneko S. Palmitate induces insulin resistance in H4IIEC3 hepatocytes through reactive oxygen species produced by mitochondria. J Biol Chem 284: 14809-14818, 2009.

28. Niess AM. Response and adaptation of skeletal muscle to exercise - the role of reactive oxygen species. Front Biosci 12: 4826-4838, 2007.

29. Nishikawa T, Edelstein D, Du XL, Yamagishi S, Matsumura T, Kaneda Y, Yorek MA, Beebe D, Oates PJ, Hammes H-P, Giardino I, and Brownlee M. Normalizing mitochondrial superoxide production blocks three pathways of hyperglycaemic damage. Nature 404: 787-790, 2000.

30. Ota T, Takamura T, Kurita S, Matsuzawa N, Kita Y, Uno M, Akahori H, Misu H, Sakurai M, Zen Y, Nakanuma Y, and Kaneko S. Insulin resistance accelerates a dietary rat model of nonalcoholic steatohepatitis. Gastroenterology 132: 282-293, 2007.

31. Otoda T, Takamura T, Misu H, Ota T, Murata S, Hayashi H, Takayama H, Kikuchi A, Kanamori T, Shima KR, Lan F, Takeda T, Kurita S, Ishikura K, Kita Y, Iwayama K, Kato KI, Uno M, Takeshita Y, Yamamoto M, Tokuyama K, Iseki S, Tanaka K, and Kaneko S. Proteasome dysfunction mediates obesity-induced endoplasmic reticulum stress and insulin resistance in the liver. Diabetes 62: 811824, 2013. 
32. Ristow M, Zarse K, Oberbach A, Kloting N, Birringer M, Kiehntopf M, Stumvoll M, Kahn CR, and Bluher M. Antioxidants prevent health-promoting effects of physical exercise in humans. Proc Natl Acad Sci 106: 8665-8670, 2009.

33. Saito $Y$. Selenoprotein $P$ as a significant regulator of pancreatic $\beta$ cell function. J Biochem 167: 119-124, 2020.

34. Speckmann B, Walter PL, Alili L, Reinehr R, Sies H, Klotz $\mathrm{LO}$, and Steinbrenner H. Selenoprotein $\mathrm{P}$ expression is controlled through interaction of the coactivator PGC- $1 \alpha$ with FoxO1a and hepatocyte nuclear factor $42 \alpha$ transcription factors. Hepatology 48: 1998-2006, 2008.

35. Stranges S, Marshall JR, Natarajan R, Donahue RP, Trevisan M, Combs GF, Cappuccio FP, Ceriello A, and Reid ME. Effects of long-term selenium supplementation on the incidence of type 2 diabetes: a randomized trial. Ann Intern Med 147: 217-223, 2007.

36. Tajima-Shirasaki N, Ishii K-A, Takayama H, Shirasaki T, Iwama H, Chikamoto K, Saito Y, Iwasaki Y, Teraguchi A, Lan F, Kikuchi A, Takeshita Y, Murao K, Matsugo S, Kaneko S, Misu H, and Takamura T. Eicosapentaenoic acid down-regulates expression of the selenoprotein $\mathrm{P}$ gene by inhibiting SREBP-1c protein independently of the AMPactivated protein kinase pathway in H4IIEC3 hepatocytes. $J$ Biol Chem 292: 10791-10800, 2017.

37. Takamura T, Misu H, Matsuzawa-Nagata N, Sakurai M, Ota T, Shimizu A, Kurita S, Takeshita Y, Ando H, Honda $\mathrm{M}$, and Kaneko S. Obesity upregulates genes involved in oxidative phosphorylation in livers of diabetic patients. Obesity 16: 2601-2609, 2008.

38. Takamura T, Misu H, Ota T, and Kaneko S. Fatty liver as a consequence and cause of insulin resistance: lessons from type 2 diabetic liver. Endocr J 59: 745-763, 2012.

39. Takamura T, Misu H, Yamashita T, and Kaneko S. SAGE application in the study of diabetes. Curr Pharm Biotechnol 9: 392-399, 2008.

40. Takamura T, Sakurai M, Ota T, Ando H, Kaneko S, and Honda M. Genes for systemic vascular complications are differentially expressed in the livers of type 2 diabetic patients. Diabetologia 47: 638-647, 2004.

41. Takayama H, Misu H, Iwama H, Chikamoto K, Saito Y, Murao K, Teraguchi A, Lan F, Kikuchi A, Saito R, Tajima N, Shirasaki T, Matsugo S, Miyamoto KI, Kaneko S, and Takamura T. Metformin suppresses expression of the selenoprotein $\mathrm{P}$ gene via an AMP-activated kinase (AMPK)/ FoxO3a pathway in H4IIEC3 hepatocytes. $J$ Biol Chem 289: 335-345, 2014.

42. Tiganis T. Reactive oxygen species and insulin resistance: the good, the bad and the ugly. Trends Pharmacol Sci 32: 82-89, 2011.
43. Ushio-Fukai M. VEGF Signaling through NADPH oxidasederived ROS. Antioxid Redox Signal 9: 731-739, 2007.

44. Veal EA, Day AM, and Morgan BA. Hydrogen peroxide sensing and signaling. Mol Cell 26: 1-4, 2007.

45. Vinceti M, Filippini T, and Rothman KJ. Selenium exposure and the risk of type 2 diabetes: a systematic review and meta-analysis. Eur J Epidemiol 33: 789-810, 2018.

46. Winterbourn CC and Hampton MB. Thiol chemistry and specificity in redox signaling. Free Radic Biol Med 45: 549-561, 2008.

Address correspondence to: Prof. Toshinari Takamura Department of Endocrinology and Metabolism Kanazawa University Graduate School of Medical Sciences 13-1 Takara-machi Kanazawa 920-8640 Japan

E-mail: ttakamura@med.kanazawa-u.ac.jp

Date of first submission to ARS Central, March 9, 2020; date of acceptance, March 18, 2020.

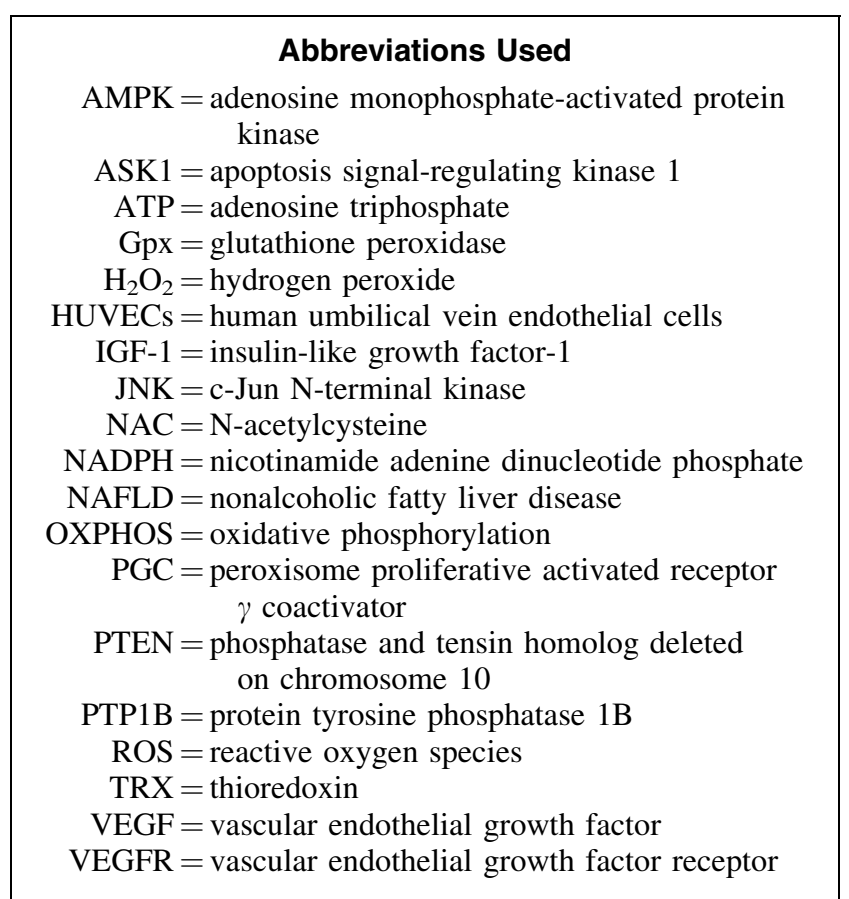

